\title{
Answer to Quiz: Echo Imaging on Page 79 and Case Discussion
}

\section{Correct answer is D - Caseous calcification of mitral valve annulus.}

Calcification of the mitral valve annulus (MVA) is a common echocardiographic finding most often seen in older female patients (1). It is usually considered as a degenerative condition. A rare variant of this pathological entity is caseous calcinosis of MVA presenting on echocardiographic imaging as a round or ovoid focal hyperechoic mass (2). It contains tooth pastelike caseous material with mixture of calcium salts, fatty acids and cholesterol (3). The liquefaction of MVA calcification is an uncommon finding happening in approximately $0.6 \%$ of patients (4). However, a large autopsy series reported that an incidence of caseous MVA calcification is $2.7 \%$. It means that this pathological entity may be mostly underrecognized (1).

Our case characterizes a typical echocardiographic appearance of caseous MVA calcification. It is a large $(2.2 \times 1.9 \mathrm{~cm})$, round, echo-dense mass containing central areas of echolucencies consistent with central liquefaction. The mass had sharp and distinct borders, with an echogenicity dissimilar from adjacent myocardium. There is no acoustic shadowing behind the mass indicative of dense calcium deposition absence.

The mechanisms involved in the liquefaction of MVA calcification are not well studied. Hypercholesterolemia and dissolution of lipidladen macrophages might be observed. High serum cholesterol level usually is associated with MVA calcification and may be related to the liquefaction necrosis. Interestingly, there is a report on a complete spontaneous resolution of a caseous calcification of the mitral annulus (5).

Caseous MVA calcification could be distinguished from commonly seen calcification by the different imaging characteristics of these two entities. When MVA calcification liquefy and becomes caseous it looks ovoid, has tumor-like appearance and mimics a mass because of its more focal appearance. In this case, there is a need to include it in differential diagnostic considerations of different intracardiac masses and tumors (6). The most reliable diagnostic method used for this purpose is cardiac computed tomography (CT) easily distinguishing the caseous MVA calcification from other heart masses by the baseline precontrast hyperattenuation with no enhancement. It is clearly seen on Figures 1 and 2 presenting cardiac $\mathrm{CT}$ examination of our patient in two different projections.

Cardiac CT picture of caseous MVA calcification obtained by unenhanced images is characteristic and CT allows a definitive diagnosis. Cardiac magnetic resonance imaging (MRI) is considered the technique of choice in the diagnosis of nontypical caseous MVA calcification. However, cardiac MRI may be inaccessible, so cardiac CT as a widely available method seems to be sufficient in addition to transthoracic echocardiography to recognize this entity.

The cardiac MRI characteristics are consistent with that of proteinaceous fluid. On MRI, calcium is generally low in signal on all sequences. However, the calcium salts and proteinaceous fluid in caseous MAC can generate high signal on $\mathrm{T} 1$ non-contrast sequences. Precontrast T1 sequences are helpful in demonstrating the distinction between MVA calcification, which have low signal on T1, and caseous MVA calcification, which demonstrate high signal on T1. Liquefied calcium is low in T2 signal intensity. Low signal was also seen on the cine SSFP sequences. Practically in all patients, the calcified shell seen also demonstrated lower signal intensity than the rest of the mass of caseous mitral annular calcification on cine SSFP. Delayed contrast enhancement typically has been reported along the periphery of caseous MVA calcification (7).

Address for Correspondence: Yuriy Ivaniv, Diagnostic Radiology Department, Danylo Halytsky Medical University, Lviv, Ukraine

Received: 15.05.2021 Revised: 07.06.2021 Accepted: 07.06.2021 


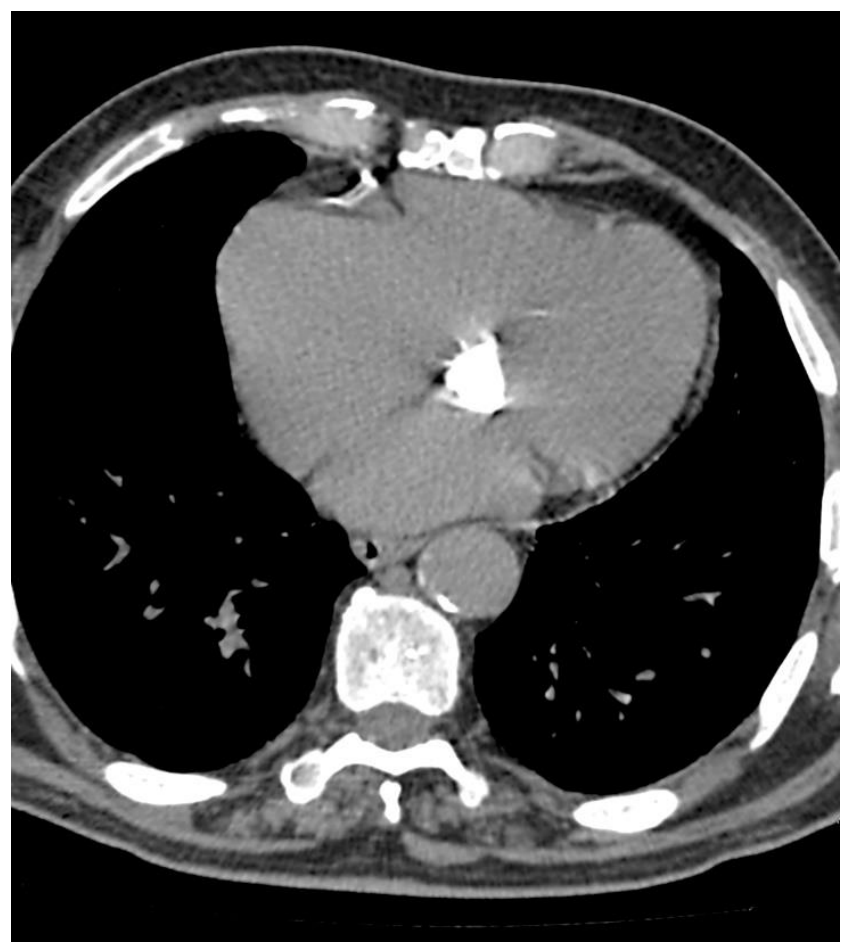

Figure 1. Axial plane computed tomography heart image

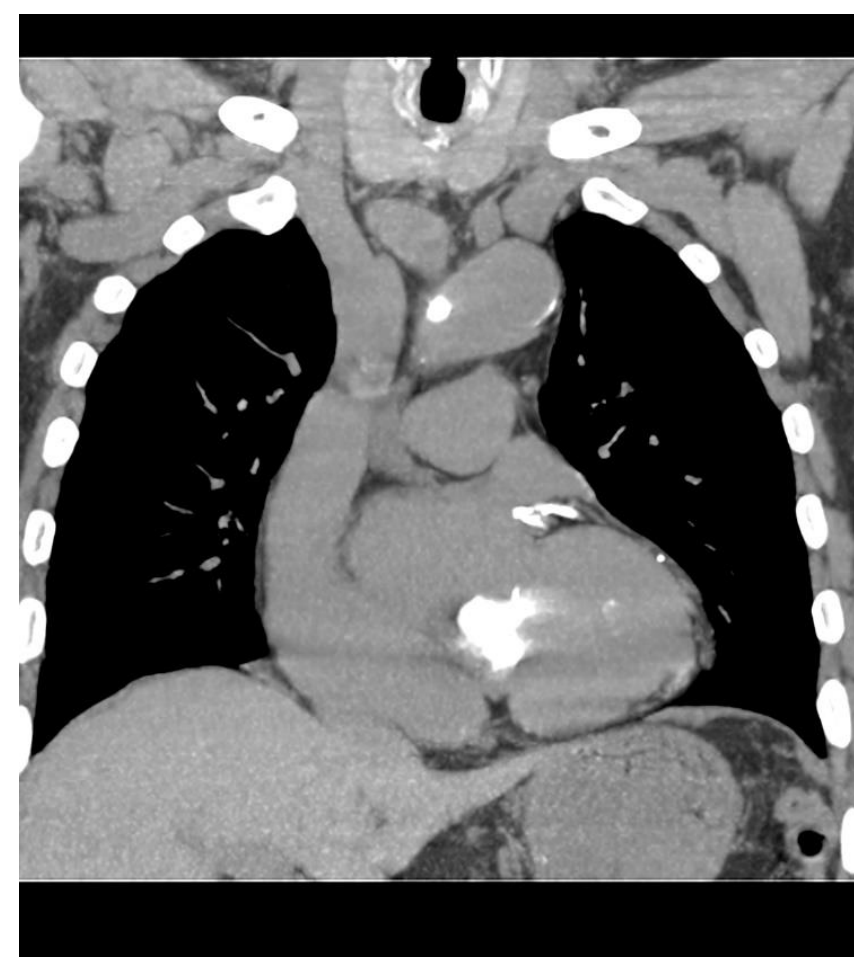

Figure 2. Coronal plane computed tomography heart image. In addition to ovoid-shaped calcification of mitral valve area, it is possible to notice the calcification of the proximal left coronary artery and its two branches. The wall of aortic arch contains calcifications too.

Despite of definite diagnosis of caseous MVA calcification based on its imaging features and typical location it may create a diagnostic dilemma. In some cases, this pathology has been misrecognized as a heart neoplasm or an abscess followed by unnecessary surgical treatment $(4,8)$. MVA calcification should be differentiated also from mitral valve calcifications itself, commonly seen on late stage of rheumatic valve disease associated with severe valve dysfunction. In such cases, calcification extends to the MVA only at the end stage of disease (9). In the contrary annular calcification is seldom associated with significant mitral valve dysfunction despite of its severity, but in some patients might be complicated by mitral regurgitation with progressive chronic heart failure (10).

Several different studies have shown convincingly that MVA calcification may be a risk factor for more significant cardiovascular pathology and associates with atrial fibrillation, conduction disorders, coronary artery disease and stroke (9).

In conclusion, it is necessary to stress that caseous MVA calcification is not a commonly recognized entity and may mimic heart masses. This type of annular calcification has a characteristic radiological appearance allowing differentiation from much often seen non-caseous type (11). It should be taken into account that the caseous MVA calcification may create diagnostic problems but familiarity with this rare entity enables accurate diagnosis and a proper management.

Yuriy Ivaniv, Natalia Lozynska, Ihor Ivaniv Diagnostic Radiology Department, Danylo Halytsky Medical University, Lviv, Ukraine

Peer-review: External and internal Conflict of interest: None to declare

Authorship: Y.I., N.L. and I.I. equally contribute to the preparation of manuscript and fulfilled all authorship criteria Acknowledgments and funding: None to declare 


\section{References}

1. Pomerance A. Pathological and clinical study of calcification of the mitral valve ring. J Clin Pathol 1970; 23: 354-61

2. Pradella S, Verna S, Addeo G, Oddo A, Miele V. Caseous calcification of the mitral annulus. J Radiol Case Rep. 2019; 13: 1-10.

3. Streian CG, Lascu A, Sosdean R, Dima CN, Grosu F, Costache A, et al. Chameleonic appearance of caseous calcification of the mitral valve - still a problem for its appropriate management. Rom J Morphol Embryol 2020; 61: 545-50.

4. Novaro GM, Griffin BP, Hammer DF. Caseous calcification of the mitral annulus: an underappreciated variant. Heart 2004; 90: 388-94. 5. Correale M, Deluca IG, leva R, Di Biase M. Spontaneous resolution of a caseous calcification of the mitral annulus. Clinics 2009; 64: 1130-2.

6. Teja K, Gibson RS, Nolan SP. Atrial extension of mitral annular calcification mimicking intracardiac tumor. Clin Cardiol 1987; 10: 546-8.

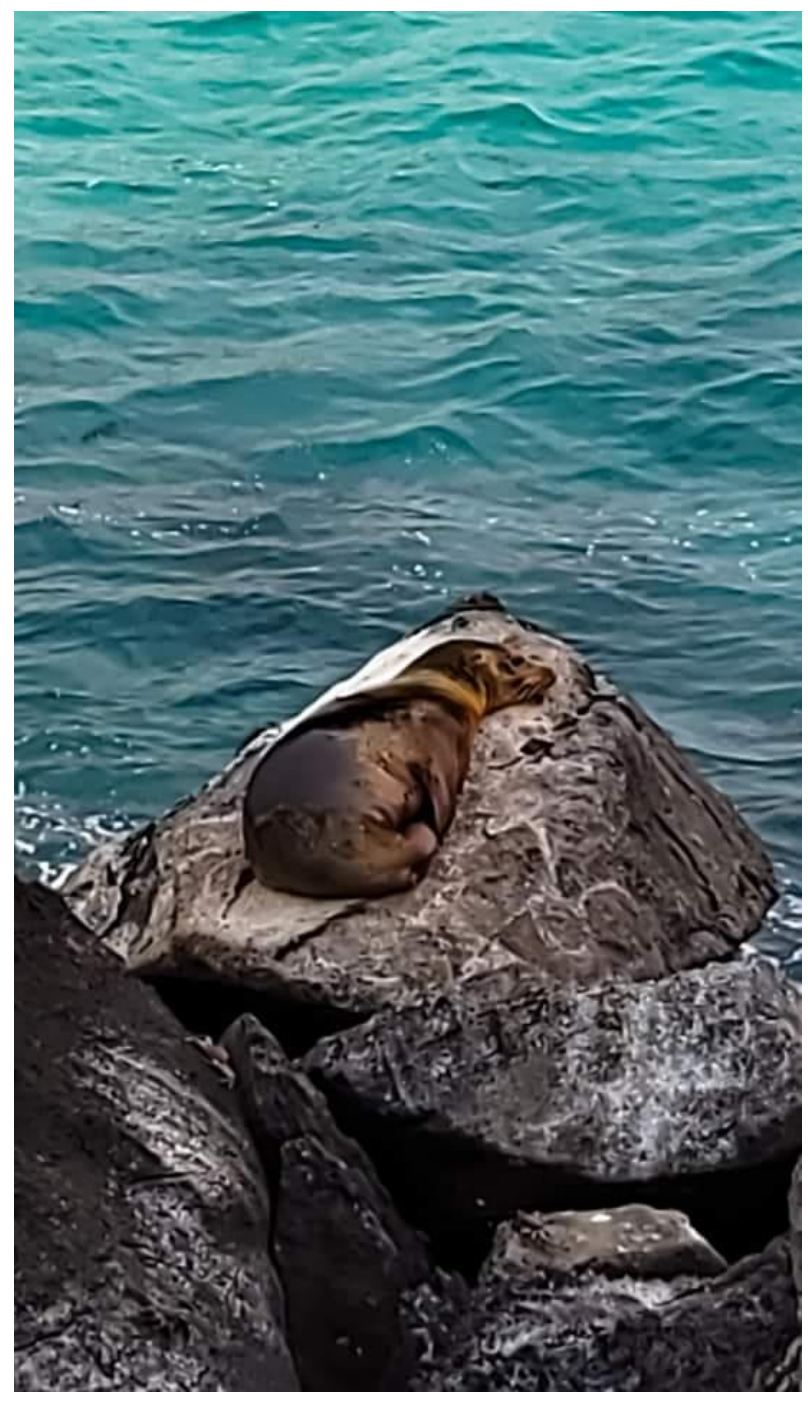

7. Di Bella G, Masci PG, Ganame J, Dymarkowski $\mathrm{S}$, Bogaert J. Images in cardiovascular medicine. Liquefaction necrosis of mitral annulus calcification: detection and characterization with cardiac magnetic resonance imaging. Circulation 2008; 117: e292-e94.

8. Curl E, Riemer E. Caseous calcification of the mitral annulus: case report and brief review. Eur Heart J Case Rep 2018; 2: yty124.

9. Shekar C, Budoff M. Calcification of the heart: mechanisms and therapeutic avenues. Expert Rev Cardiovasc Ther 2018; 16: 527-36.

10. Pala AA, Iner H, Ercisli MA. Approach to an unusual cardiac mass: mitral annulus caseoma. Braz J Cardiovasc Surg 2020; 35: 120-2.

11. Michałowska I, Szymanski P, Kwiatek P, Spalek $M$, Furmanek $M$, Zielinski $P$, et al. Caseous calcification of the mitral annulus - the complementary role of computed tomography and transthoracic echocardiogram. Pol J Radiol 2018; 83: e621-6. 